\title{
Etude de la répartition spatiale des effets du transport de neige par le vent; premiers travaux réalisés à partir de mesures systématiques sur le terrain
}

\author{
M. Mases L. Buisson \\ Division Nivologie Cemagref \\ W. Good \\ IFENA \\ J. M. Vilaplana \\ Universitat de Barcelona
}

\begin{abstract}
Les problèmes liés à la répartition irrégulière de la neige transportée par le vent sont bien connus. L'accumulation de la neige par le vent joue un rôle dans le déclenchement des avalanches. Elle peut également créer des entraves à la circulation sur des axes routiers ou des voies ferrées. L'érosion du manteau neigeux par le vent peut aussi dégrader les pistes de ski et être à l'origine de pertes d'exploitation importantes dans les stations de ski. Les conséquences hydrologiques sont aussi importantes parce que la neige transportée par le vent peut déterminer la quantité d'eau de l'aquifère d'un bassin ; il ne faut pas oublier que la neige constitue une importante ressource en eau. $\mathrm{Ce}$ thème $\mathrm{a}$, depuis longtemps, suscité des études. Nous pouvons citer les travaux réalisés par Tabler [1], Föhn [2], Meister [3], Castelle [4], Naaim [5].

Il est donc évident que connaître la répartition spatiale de la neige transportée par le vent peut aider à résoudre de nombreux problèmes dans le domaine des risques naturels et, par ailleurs, une meilleure connaissance des effets spatiaux du
\end{abstract}

transport de neige par le vent peut être utile en hydrologie pour une meilleure estimation de la ressource en eau.

Dans le cadre d'un projet européen consacré à l'étude des avalanches, nous avons abordé le sujet du transport de neige par le vent à travers l'exploitation de données nivo-météorologiques recueillies sur un site suisse: le Stillberg (fig. 1).

Le but de notre travail est d'étudier la distribution spatiale de la neige transportée par le vent, dans une zone de départ d'avalanches en haute montagne. Il n'est pas exclu que les résultats de cette étude puissent être utilisés pour d'autres contextes d'utilisation.

De façon plus précise, nous souhaitons, dans un premier temps, comprendre l'effet de la topographie sur cette répartition. Dans un deuxième temps, nous essayons, grâce aux données de terrain et à une meilleure compréhension de cet effet, de valider et d'améliorer un modèle de transport de neige par le vent existant au sein du système ELSA [6].

\section{Space distribution study of the effects of wind snow transport}

This paper deals with the beginning of a research project dedicated to the study of snow distribution caused by wind. This project is based on the analysis of snow depths and meteorological data. These data have been collected on a Swiss site: The Stillberg.

The measurement collection started in 1958. The first twelve years have been analysed. The analysis has begun with the selection of the snow transport events with strong and regular winds. Then, for each event, a map of snow balance and snow depth profiles have been produced.

These graphic documents show the location of zones with suitable conditions for drifted snow to be accumulated. 




1. Situation géographique du site du Stillberg.

Jusqu'à maintenant, seul a été réalisé un traitement spatial des données de hauteur de neige du Stillberg, car nous ne disposons pas encore des données météorologiques relevées sur site. Nous avons donc utilisé les données météorologiques d'une station proche située sur le Weissfluhjoch à $6 \mathrm{~km}$ au nord-ouest.

\section{LE SITE ET LES DONNÉES DU STILL- BERG}

Le site du Stillberg est un versant qui se trouve au sud-est de la ville de Davos, dans la vallée de la Dischma, au cœur du canton des Grisons (fig. l). Il s'agit d'un versant exposé au nord-est qui possède une zone de déclenchement caractéristique, située à la limite supérieure de la forêt entre 2000 et $2250 \mathrm{~m}$ d'altitude. Le site du Stillberg est constitué d'une alternance d'arêtes et de talwegs. Dans certains cas, ces arêtes provoquent l'accumulation de la neige sous le vent.

A l'origine des mesures (à partir de 1958), les recherches conduites par l'IFENA (Institut Fédéral pour l'Etude de la Neige et des Avalanches) étaient orientées vers l'élaboration d'une méthode de reboisement dans une zone de déclenchement d'avalanches. Dans ce contexte, il y a eu, sur le versant, mise en place de deux réseaux de perches pour mesurer la hauteur de neige et d'une série de capteurs de données nivo-météorologiques. Des photographies ont été prises depuis le versant d'en face. La distribution dans le temps des relevés est présentée ci-dessous (fig. 2) :

C'est donc une série unique et extraordinaire de données, presque 36 années d'enregistrement ! Dans un premier temps, nous avons décidé d'analyser les douze premières années de mesures nivologiques au Stillberg (mesures des hauteurs de neige) et les mesures météoro-



2. Répartition temporelle des données du Stillberg. On voit apparaître en grisé les données utilisées jusqu’à maintenant. 
logiques du Weissfluhjoch contenues dans le rapport « Schnee und Lawinen in den Schweizer Alpen " publié chaque année par l'IFENA.

Nous présentons ici un résumé de la première analyse des données et des premières observations sur la distribution spatiale de la neige.

\section{II $\square$ SÉLECTION DES ÉPISODES DE TRANS- PORT DE NEIGE}

On peut définir, en premier lieu, un intervalle de mesure comme une période de temps limitée par une mesure de hauteur de neige au début et d'une autre à la fin.

La hauteur de neige est mesurée avec l'observation des 370 perches mises en place sur tout le versant (fig. 3). Dans les 12 premières années de données de hauteur de neige, il y a 418 intervalles de mesure. Evidemment ces intervalles ne contiennent pas tous des périodes de transport de neige.

Il faut les sélectionner. La sélection des intervalles a été réalisée, d'une part, à partir des données de hauteur de neige au Stillberg et, d'autre part, à partir des données météorologiques au Weissfluhjoch. Ensuite nous avons comparé ces deux extractions et nous avons fait une sélection définitive des intervalles qui sont intéressants pour l'étude (ils contiennent les périodes de transport de neige). Ces intervalles constituent des épisodes de transport de neige.

Les critères de sélection relatifs aux hauteurs de neige ont été les suivants :

- On cherche les intervalles où la hauteur de neige augmente ou bien diminue considérablement.

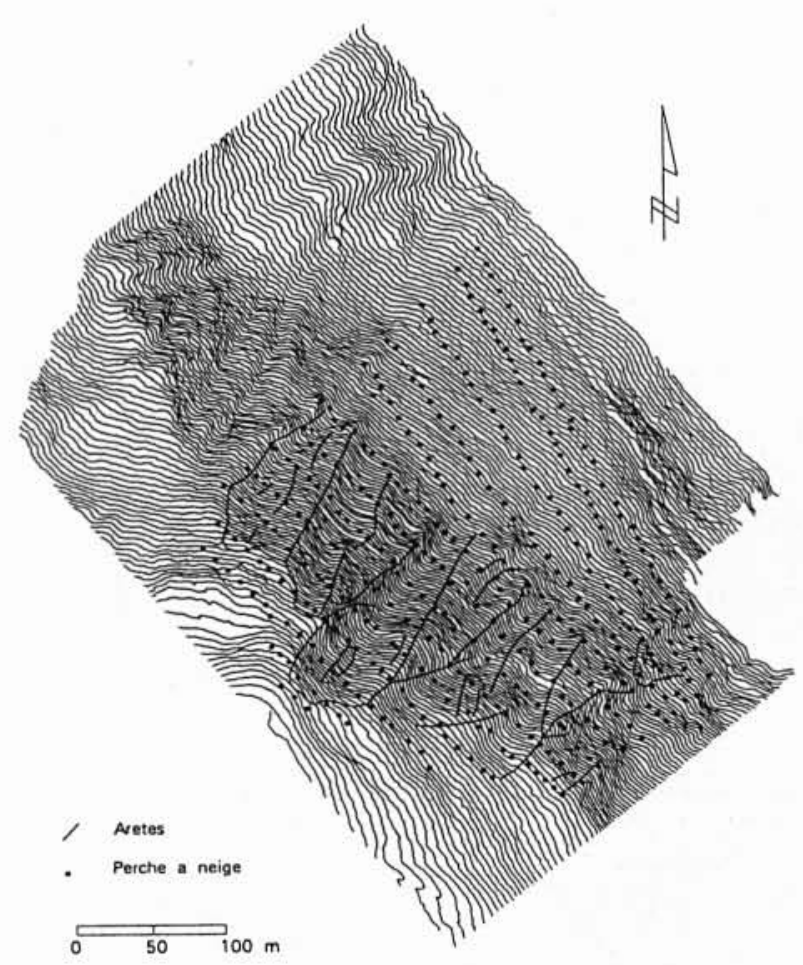

3. Réseau de perches à neige au site de Stillberg.
- On cherche les intervalles où l'écart-type des valeurs de hauteur de neige au niveau des perches est important ou a une grande variabilité du début à la fin de l'intervalle. On s'aperçoit, en effet, que les périodes de fusion ou les périodes d'enneigement sans vent font varier la hauteur moyenne de la neige (maximale et minimale) mais ne font pas varier l'écart-type.

- Un critère proche du précédent consiste à vérifier si la hauteur minimale de la neige varie et si la hauteur maximale ne varie pas ou bien le contraire. Cela veut dire que les processus d'accumulation et d'érosion ne sont pas uniformes, situation caractéristique des épisodes de transport.

Les critères de sélection relatifs aux données métérologiques sont la précipitation (plus de $10 \mathrm{~cm}$ ont été considérés suffisants pour donner des accumulations importantes avec un fort vent) ainsi que, évidemment, la vitesse et la direction du vent. Les intervalles ont été sélectionnés s'ils ont des rafales de vent supérieures à $6 \mathrm{~m} / \mathrm{s}$ et si le vent maintient une direction presque constante.

La comparaison des données de hauteur de neige et des données métérologiques permet d'identifier les meilleurs épisodes de transport pour l'étude.

\section{TRAITEMENT DES DONNÉES DE HAUTEUR DE LA NEIGE}

Ce qui nous intéresse, en premier lieu, c'est de voir l'effet d'un épisode de vent dans le secteur d'étude. Quelle est la

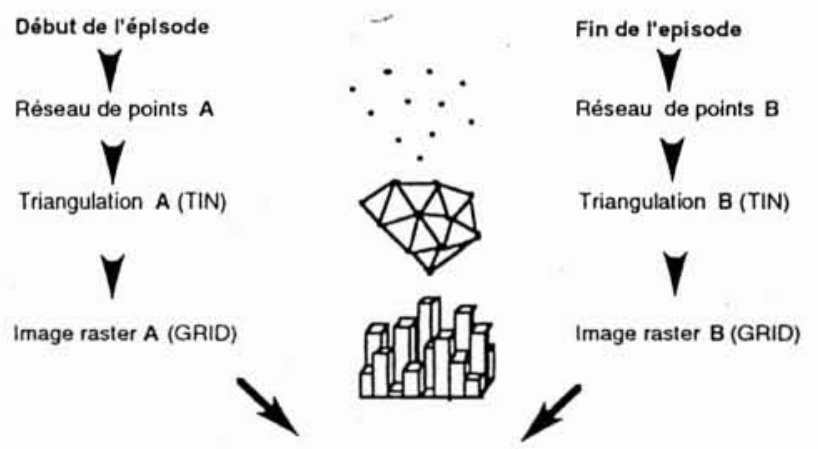

Image $\mathrm{B} \cdot$ Image $\mathrm{A}=$ Image $\mathrm{C}$

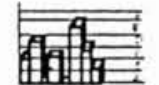

Définition d'intervalles

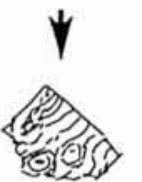

Carte de bilan de neige

4. Procédure suivie pour le traitement des données d'épaisseur de la neige du Stillberg. TIN et GRID sont deux des modules d'ARC-INFO. 


\section{RÉPARTITION DES EFFETS DU TRANSPORT DE NEIGE PAR LE VENT}

répartition spatiale de la neige ? Où se trouvent les suraccumulations et les zones balayées, qui restent sans neige, après les vents forts ?

La procédure consiste à réaliser des cartes de bilan de neige. Pour la confection de ces cartes, le SIG (Système d'Information Géographique) Arc-Info a été utilisé.

On part de deux groupes de données ponctuelles: les données de hauteur de neige au début de l'épisode et les données de hauteur de neige à la fin de l'épisode. Pour chaque groupe, une triangulation des hauteurs de neige a été faite. Cette triangulation a ensuite été transformée en une image « raster» qui permet de faire facilement des calculs.

Une troisième image raster a été obtenue à partir de la soustraction de l'image correspondante au début de l'épisode à celle de la fin. Cette image est déjà une image du bilan de neige. On peut obtenir la carte de bilan de neige définissant des classes en dessinant des isolignes correspondant à ces classes (fig. 4).

\section{Q OBSERVATION DES CARTES DE BI- LAN DE NEIGE}

Les directions du vent qui provoquent les accumulations les plus régulières et les plus constantes sont les directions N-NW-NNW et S-SE-SSE. Ces deux directions du vent sont presque perpendiculaires à la direction moyenne des arêtes du site du Stillberg. Il va sans dire que dans ces deux directions les arêtes ont la meilleure efficacité.

De l'observation des cartes de bilan de neige (fig. 5), on retient certaines caractéristiques de la distribution spatiale de la neige :

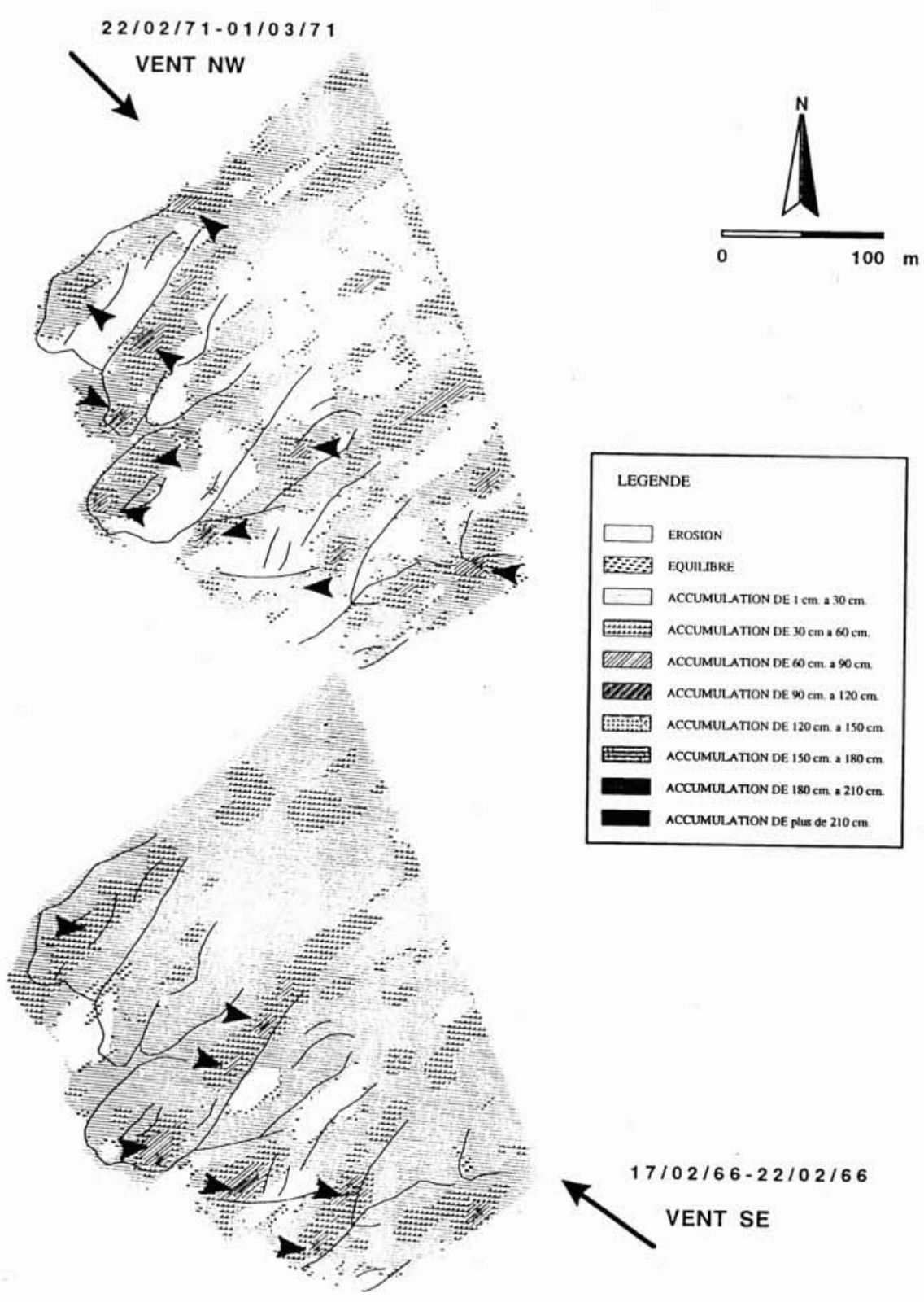

5. Cartes de bilan de neige. Les flèches indiquent les endroits d'accumulation préférentielle. 
- Les accumulations et les zones d'ablation sont de caractère linéaire et longent les arêtes.

- Il y a des zones d'accumulation préférentielles (dans les deux directions étudiées) qui se répètent à chaque épisode. Elles sont identifiées par des flèches.

- Si on compare les zones d'accumulation préférentielle avec la pente du versant ( $f i g .6$ ), on ne voit, a priori, aucune relation entre la pente et la présence d'une accumulation. Certaines coïncident avec des pentes importantes (supérieures à 40 degrés) probablement avec une accumulation en forme de corniche, mais d'autres se situent en zones moins raides.



6. Carte des pentes du Stillberg.

\section{DLES PROFILS DE HAUTEUR DE NEIGE}

Le fait que les accumulations les plus grandes se produisent toujours dans les mêmes endroits, de façon grossièrement indépendante de la vitesse du vent et de la qualité de la neige en surface, nous permet de penser que la topographie joue un rôle déterminant dans la distribution des accumulations. On a déjà vu que la pente ne jouait pas un rôle déterminant. Cependant, la pente mesurée sur un profil perpendiculaire à l'arête joue probablement, elle, un rôle plus important.

En tentant d'approfondir cette analyse, nous avons réalisé des profils perpendiculaires aux arêtes, dans la direction des vents dominants. Dans la suite de cette commu- nication, les expressions relatives à la pente («pente ", « raide ", « horizontale») font référence aux pentes de ces profils. Nous présentons ici deux séries de profils de l'année 1964 (fig. 7).

Ces profils mettent en évidence certaines caractéristiques :

- Les profils topographiques et de hauteur de neige correspondent à la pente dans la direction du vent dominant. Dans ces conditions, on voit parfaitement que les accumulations principales de neige ont lieu, par rapport aux autres, dans les versants sous le vent les plus horizontaux dans cette direction $\left(\leq 20^{\circ}\right)$.

- Au début de la saison, les versants qui sont remplis de neige en premier sont aussi les versants les moins raides. En suivant l'idée de [1], ce sont les premiers versants à s'approcher du profil d'équilibre. Les versants les plus raides $\left(\geq 30^{\circ}\right)$ sont remplis plus tard et par des vents très forts.

- Il n'est pas nécessaire que le versant soit à l'abri du vent pour que se développe une accumulation. A partir des données extraites des profils du Stillberg, on observe qu'une rupture de pente de 10 degrés est suffisante pour engendrer une accumulation de neige sur le versant au vent. Dans la figure 8, la pente ne change que de 19 à 9 degrés et pourtant cette morphologie provoque l'accumulation de la neige.

- La plupart des arêtes, dans la partie la plus haute du site, au vent, ont une zone très balayée, sans neige. L'accumulation de la neige débute après cette zone arrivant au maximum dans le versant aval (fig. 8).

Si les sur-accumulations de neige les plus importantes ont lieu dans les parties du versant les plus horizontales, suivant la direction du vent, on peut s'attendre à ce que les zones les "plus dangereuses ", c'est-à-dire avec le plus d'accumulation de neige susceptible d'être instable, soient celles qui présentent un profil transversal, parallèle au vent, peu raide et un profil longitudinal très raide.

\section{VI $\square$ CONCLUSIONS ET PERSPECTIVES}

Dans le site du Stillberg, les vents qui provoquent les accumulations les plus régulières et les plus constantes sont les vents qui soufflent dans les directions perpendiculaires à celles des arêtes (N-NW et S-SE).

Les accumulations se produisent toujours dans les mêmes endroits, avec une morphologie linéaire parallèle aux arêtes.

Les zones d'accumulation et d'érosion sont déterminées par la topographie locale, essentiellement par la pente du versant perpendiculaire à la direction de l'arête. En revanche, la pente longitudinale du versant ne joue pas un rôle déterminant dans la distribution des accumulations.

En général, les pentes transversales les moins raides (moins d'environ 20 degrés) provoquent les accumulations les plus importantes.

En poursuivant ces analyses qui pourront sans doute être précisées grâce aux données nivo-météorologiques relevées sur le site du Stillberg lui-même et que nous n'avons pu encore exploiter, nous commençons actuellement à valider et à améliorer un modèle de transport de neige par le vent existant au sein du système ELSA. 

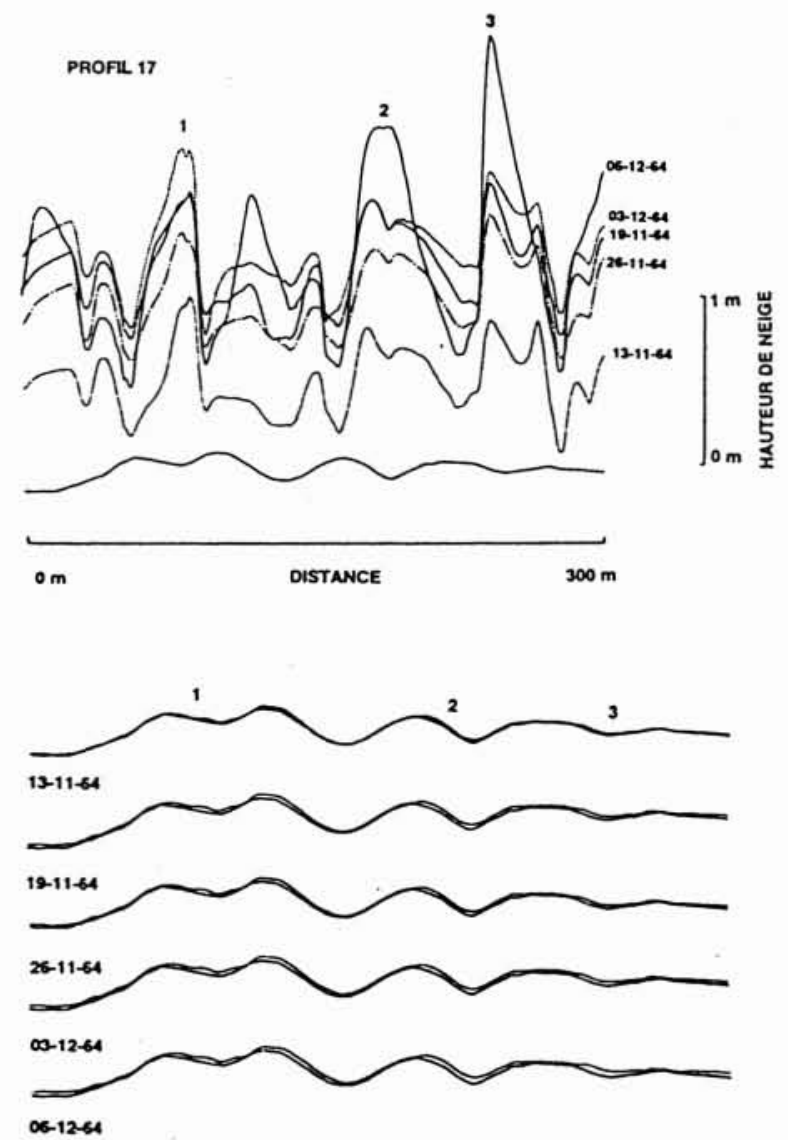

7. Profils de hauteur de neige perpendiculaires aux arêtes. Dans la partie supérieure, l'échelle de la hauteur de neige a été exagérée, dans la partie inférieure, l'échelle est réelle.

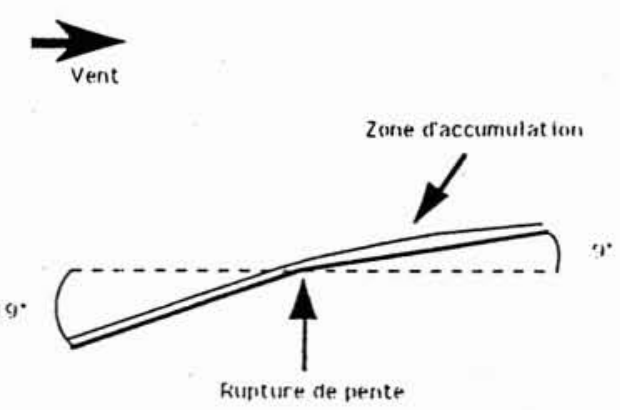

8. Représentation de deux des endroits typiques où il y a des accumulations et des zones balayées dans le site du Stillberg.

\section{Références}

[1] TABLER R. (1975). - Predicting profiles of snowdrifts in topographic catchments. West Snow Conference. Coronado California. Proc. 43, pp. 87-97.
[2] FÖHN P., MEISTER R. (1983). - Distribution of snow drifts on ridge slopes: measurements and theoerical approximations. Annals of Glaciology, 4, pp. 52-57.

[3] MEISTER R. (1989). - Influence of strong winds on snow distribution and avalanche activity. Annals of glaciology, 13, pp. 195-201.

[4] CASTelle T. (1991). - Utilisation des moyens photogramétriques pour la saisie de l'évolution de dépôts de neige sur un site. Symposium de Chamonix CISA-IKAR, Chamonix.

[5] NaAim F., Brugnot G. (1992). - Transport de la neige par le vent. Connaissances de base et recommandations. CEMAGREF / Division de Nivologie. ISBN 2-85362-312-2.

[6] Buisson L., Charlier C. (1993). - Avalanche modelling and integration of expert knowledge in the ELSA system. Annals of Glaciology, 18, 123-128. 